\title{
Modeling electro-optical response of nematic liquid crystals by numerical methods
}

\section{Modelado de la respuesta electro-óptica del cristal líquido nemático mediante métodos numéricos}

\author{
J. F. Algorri $\left.{ }^{*}\right)$, V. Urruchi, P. J. Pinzón, J. M. Sánchez-Pena \\ Departamento de Tecnología Electrónica, Universidad Carlos III, Butarque 15, E28911 Leganés, Madrid, Spain. \\ (*) Email: jalgorri@ing.uc3m.es \\ Received / Recibido: 05/08/2013. Revised / Revisado: 08/10/2013. Accepted / Aceptado: 28/10/2013. \\ DOI: http://dx.doi.org/10.7149/OPA.46.4.327
}

\begin{abstract}
:
In this study, a novel algorithm to solve the position of a nematic liquid crystal molecular director inside a device has been proposed. The formulation for minimizing the Gibbs free energy, composed by the free energy deformation (Frank-Oseen equations) minus the electrical energy, has been established using a vectorial representation of the director. The three liquid crystal elastic constants have been considered. The differential equations involved have been solved by applying numerical methods, using an algorithm developed by the authors. It has been compared with other techniques and the main advantages are that the new algorithm is faster and more accurate than other previous methods such as finite difference method (FDT) or a boundary value problem solver of MATLAB (bvp4c). Simulation program is also a powerful tool for analyzing other liquid crystal properties such as refractive index and permittivity as a function of voltage. To validate the simulation results, they have been compared with some measurements of a nematic liquid crystal device manufactured for that purpose. The comparison of birefringences simulated and measured show results that are fairly in agreement. This work also aims to provide a software simulation tool easy to reprogram for more complex devices.
\end{abstract}

Key words: Optoelectronics; Liquid Crystals; Liquid-Crystal Devices.

\section{RESUMEN:}

En este trabajo se propone un nuevo algoritmo para resolver la posición molecular del cristal liquido nemático (CLN) en un dispositivo afectado por un campo eléctrico. La formulación característica del CLN bajo la influencia de un campo eléctrico se compone de la energía libre de deformación de Frank-Oseen menos la energía eléctrica. Este resultado, considerado como energía libre de Gibbs, tiene un mínimo cuando el director molecular se encuentra en equilibrio. La minimización de esta energía ha sido establecida usando una representación esférica del director molecular. Se han considerado las constantes elásticas del CLN y se ha resuelto mediante métodos numéricos. Para ello se emplea un algoritmo desarrollado por los autores, que ha sido comparado con otros métodos propuestos en la literatura como el método de las diferencias finitas (FDT) o un método de resolución propietario de MATLAB conocido como bvp4c. Las comparativas realizadas muestran un comportamiento superior del algoritmo propuesto, siendo más rápido y preciso que los métodos anteriores. Una vez que se conoce la posición de las moléculas en el dispositivo, otros parámetros como índice de refracción y permitividad en función del voltaje, pueden ser determinados. Para dar una validez a los resultados de simulación, se ha fabricado y caracterizado un dispositivo monopixel de CLN. Las medidas experimentales de birrefringencia y permitividad para diferentes tensiones de alimentación muestran una gran concordancia con los resultados de simulación. Este trabajo pretende proporcionar una herramienta de simulación que puede emplearse en otros dispositivos de CLN más complejos en los cuales se conoce la distribución de tensión.

Palabras clave: Optoelectrónica; Cristal Líquido; Dispositivos de Cristal Líquido. 


\section{REFERENCES AND LINKS / REFERENCIAS Y ENLACES}

[1]. I. Abdulhalim, D. Menashe, "Approximate analytic solutions for the director profile of homogeneously aligned nematic liquid crystals", Liq. Cryst. 37, 233-239 (2010). DOI

[2]. P. A. Cruz, M. F. Tomé, S. McKee, I. W. Stewart, "Numerical simulation of director orientation of nematic liquid crystal in tumbling flows", V European Conference on Computational Fluid Dynamics, ECCOMAS CFD (2010).

[3]. B. F. de Oliveira, P. P. Avelino, F. Moraes, J. C. R. E. Oliveira, "Nematic liquid crystal dynamics under applied electric fields", Phys. Rev. E 82, 041707 (2010). DOI

[4]. S. Craig, C. S. MacDonald, J. A. Mackenzie, A. Ramage, C. J. P. Newton, "Robust adaptive computation of a one-dimensional Q-tensor model of nematic liquid crystals", Comput. Math. Appl. 64 3627-3640 (2012). DOI

[5]. C. W. Oseen, "Beiträge zur theorie anisotroper flüssigkeiten", Ark. Math. Fys. 19A, 1-19 (1925).

[6]. H. Zocher, "Ber die einwirkung magnetischer, electrischer und mechanischer kräfte auf mesophasen", PhysZ 28, 790-796 (1927).

[7]. F. C. Frank, "On the theory of liquid crystals", Discuss. Faraday Soc. 25, 19-28 (1958). DOI

[8]. C. W. Oseen, "The theory of liquid crystals", Trans. Faraday Soc. 29, 883-900 (1933). DOI

[9]. J. L. Ericksen, “Conservation laws for liquid crystals", J. Rheology 5, 23-34 (1961). DOI

[10]. F. M. Leslie, "Some constitutive equations for liquid crystals", Arch. Rational Mech. AMI. 28, 265-283 (1968).

[11]. H. Mori, E. C. Gartland Jr., J. R. Kelly, P. J. Bos, "Multidimensional director modeling using the Q tensor representation in a liquid crystal cell and its application to the $\pi$ cell with patterned electrodes", Jpn. J. Appl. Phys. 38, 135-146 (1999). DOI

[12].Z. Ge, T. X. Wu, R. Lu, X. Zhu, H. Qi, S.-T. Wu, “Comprehensive three-dimensional dynamic modeling of liquid crystal devices using finite element method", J. Displ. Technol. 1, 194-206 (2005). DOI

[13]. P. Vanbrabant, R. James, J. Beeckman, K. Neyts, E. Willman, F. A. Fernandez, “Three-dimensional finite element modeling of liquid crystal devices", Proc. SPIE 7955, 79550B (2011). DOI

[14]. S. L. Subota, V. Y. Reshetnyak, S. P. Pavliuchenko, T. J. Sluckin, "Numerical modeling of tunable liquidcrystal-polymer-network lens", Mol. Cryst. Liq. Cryst. 489, 40-53 (2008). DOI

[15]. I. Abdulhalim, D. Menashe, "Approximate analytic solutions for the director profile of homogeneously aligned nematic liquid crystals", Liq. Cryst. 37, 233-239 (2010). DOI

[16]. L. J. B. Márquez, "Solución numérica de problemas de valor frontera para ecuaciones diferenciales ordinarias", PhD Thesis, Universidad de los Andes (2010).

[17]. J. Kierzenka, L. F. Shampine, "A BVP solver that controls residual and error", J. Numer. Anal. Indust. Appl. Math 3, 27-41 (2008).

[18]. C. C. Wang, M. J. Jang, P. L. Ko, “A measurement system for the phase retardation of liquid crystal particles under an electric field effect”, J. Phys. - Conf. Series 48, 957-969 (2006).

[19]. A. K. Kirby, P. J. W. Hands, G. D. Love, "Optical design of liquid crystal lenses: Off-axis modeling”, Proc. SPIE 5874, 587407 (2005). DOI

[20].V. Urruchi, I. Pérez, N. Gaona, J. M. Sánchez-Pena, "Phase modulation and optical anisotropy of high birefringence liquid crystals", Mol. Cryst. Liq. Cryst. 502, 207-219 (2009). DOI

\section{Introduction}

Knowledge of the nematic LC (NLC) molecular directions inside a device is essential for predicting the electro-optic response as a function of external stimulus. Some recent applications such as optical filters, microwave phase shifters and filters, active zoom, and tunable lenses, among others, have been areas of interest for NLC simulations. Recently, some studies have tried to solve this problem analytically [1]. However, in order to obtain accurate results, numerical methods are required. Recent numerical simulations have focused on NLC in tumbling flows [2], nematic dynamics [3], and new algorithms [4]. The objective of these works is to find the position of 
the NLC director inside the cell. In our study we propose a novel algorithm to solve the director equations under applied electric fields.

In the NLC physics understanding, the most accepted theory is the continuum theory. In this theory the average local orientation of molecules is mathematically represented by a vector called director $\vec{n}$. All directors that form a LC cell determine the anisotropy inside it. This vector can be represented in Cartesian $\left(n_{x}, n_{y}, n_{z}\right)$ or spherical coordinates with $r=1(\theta, \varphi)$, see Fig. 1. The equilibrium state is such that all the molecules are aligned parallel to each other in the direction of $\vec{n}$. However, as a result of the external fields, this director is deformed. The continuum theory does not consider the NLC behaviour at a structural level because the lengths of these deformations (about $1 \mu \mathrm{m}$ ) are considerably shorter than the molecular length (Armstrong). The basis for the continuum theory model was proposed at the end of 1920's by C. W. Oseen [5] and H. Zöcher [6]. The first one developed a NLC static theory; the second one successfully applied this theory to the Freedericksz transition. However, it was not completed until over thirty years later when F. C. Frank studied this theory and presented it as elasticity deformation theory [7], offering a direct formulation of the energy. These equations are usually employed in the static director simulations. The dynamic theory is attributed to Oseen [8], the formulation of general conservative laws to Erickseen [9] and the constitutive equations describing the mechanical behaviour to Leslie [10]; in fact, these equations are easily applicable to

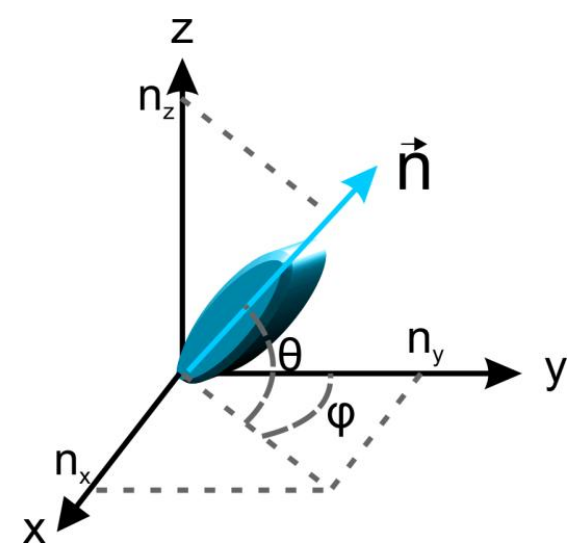

Fig. 1. Molecular director of a LC molecule, Cartesian and spherical coordinates. numerical simulations of the LC director dynamics. These theories are based on the study of two parameters: the $Q$ tensor, which is related with S parameter (order parameter) [11] or director $\vec{n}$. The molecular director $\vec{n}$ is more frequently used due to its mathematical simplicity, low computational cost and more accurate results [12].

Until now different approaches have been proposed to minimizing the free Gibbs energy, which is composed by the electrical energy plus free energy deformation (Frank-Oseen equations). These methods are based on EulerLagrange equations. To solve these equations, one of the most employed techniques is the finite element method [13], this method is usually employed in complex structures; its programming involves a variational approach to the Frank-Oseen free energy formulation. The method also needs mesh grids that usually requires many lines of code. Another method is to solve the equations directly, employing numerical methods to solve the resulting differential equations. Several numerical methods have been proposed to solve this problem, finite difference [14], Bvp4c [15], etc. The finite difference method is a simple method but has to be precisely configured in order to work properly, the step constant is usually a difficult value to find (small values causes high computational costs and big values big errors). The Bvp4c is a MATLAB function; it is timeconsuming but the less erroneous [16], actually there have been advances on the algorithm through reduction in errors [17] but has more difficulties to converge. The main differences between them are the error and the computational cost. The aim of this work is to provide an easy tool to solve the molecular distribution. A simple, fast and reliable algorithm is proposed to solve these equations.

\section{Solving continuum theory}

Molecular alignment of a LC is deformed under mechanical stress. In nematic LC, three deformations can be considered: splay, twist and bend.

The relation between deformation and stress, for each kind of deformation, is expressed as 
splay elasticity, $K_{11}$, twist elasticity, $K_{22}$ and bend elasticity, $K_{33}$, as shown in Fig. 2. The elastic constants for a LC are around $10^{-6}, 10^{-7}$ dynes and therefore, much smaller than common solids. This fact makes modifications of molecules positions easy, due to electrical or magnetic external fields. Mathematically, if an external perturbation, such as an electrical field, is introduced in the system, the deformation of the molecular director can be estimated minimizing the Gibbs free energy density, $F_{G}$. This energy is composed of the contribution of two energies: the deformation free energy, $F_{d}$, and the electrostatic free energy, $F_{e}$, $\left(F_{G}=F_{d}-F_{e}\right) . F_{d}$ is the energy of the different deformation modes, as a function of the gradient of $\vec{n}$, and is determined by the free energy formulation of Frank-Oseen:

$$
\begin{gathered}
F_{d}=K_{11}(\nabla \vec{n})^{2}+K_{22}\left(\vec{n} \cdot \nabla \times \vec{n}+\frac{2 \pi}{\xi}\right)^{2}+ \\
+K_{33}|\vec{n} \cdot \nabla \times \vec{n}|^{2},
\end{gathered}
$$

where $\vec{n}$ is the molecular director, $\vec{n}=$ $\left(n_{x}, n_{y}, n_{z}\right), K_{i i}$ are the elastic deformation constants and $\xi$ is the pitch of a quiral helicoidal dopant (for twist purposes); in the simulation program this variable is called $P$. On the other hand, $F_{e}$ is given by:

$$
F_{e}=-\frac{1}{2} \vec{D} \cdot \vec{E},
$$

following the Maxwell equations:

$$
\vec{E}=-\nabla V, \quad \vec{D}=\overline{\bar{\varepsilon}} \vec{E},
$$

where the dielectric tensor for a nematic LC is:

$$
\overline{\bar{\varepsilon}}=\left[\begin{array}{ccc}
\varepsilon_{0}+\Delta \varepsilon n_{x}^{2} & \Delta \varepsilon n_{x} n_{y} & \Delta \varepsilon n_{x} n_{z} \\
\Delta \varepsilon n_{y} n_{x} & \varepsilon_{0}+\Delta \varepsilon n_{y}^{2} & \Delta \varepsilon n_{y} n_{z} \\
\Delta \varepsilon n_{z} n_{x} & \Delta \varepsilon n_{z} n_{y} & \varepsilon_{0}+\Delta \varepsilon n_{z}^{2}
\end{array}\right],
$$

$\Delta \varepsilon$ is the dielectric anisotropy and $\varepsilon_{0}$ the ordinary permittivity. The Gibbs free energy (WG), given by the integral of the free energy density $\left(F_{G}\right)$ over the thickness, has a minimum in the equilibrium state. However, due to the high nonlinearity of the free energy and the

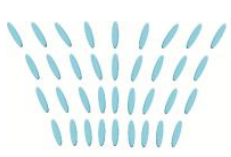

a)

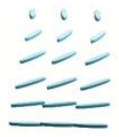

b)

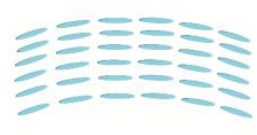

c)
Fig. 2. Deformations in nematics: a) Splay, b) twist, c) bend. coupling between deformation and electrical energies, a direct solution of the LC director deformation from the free energy equation is very difficult to obtain. Therefore, the modelling usually involves an iterative process, minimizing the Gibbs free energy to update the LC director profile. Also, the electric energy is minimized by solving Gauss's Law to up-date the potential profile interactively.

\section{2.a. Isolating variables}

In order to simplify the problem, the molecular director orientation is described with two angles: $\theta$, defined as the director tilt angle measured from the device surface, and $\varphi$, defined as the twist angle measured from the alignment direction (See Fig. 1):

$$
\vec{n}=(\cos \theta \cos \varphi, \cos \theta \sin \varphi, \sin \theta) .
$$

With this simplification, considering Eq. 2, and one dimension, the electrostatic free energy results:

$$
F_{e}=-\frac{1}{2}\left(\varepsilon_{0}-\Delta \varepsilon \sin (\theta(z))\right)\left(\frac{\partial V}{\partial z}\right)^{2} .
$$

At this point, and considering the Gibbs free energy as:

$$
W_{G}=\frac{1}{2} \int_{0}^{d}\left(F_{d}-F_{e}\right) d z
$$

where $d$ is the LC thickness, the minimum energy solution (subject to boundary conditions), produced in the nematic equilibrium state, is equivalent to solve the Euler-Lagrange equations. Those equations, which are in one dimension and spherical coordinates, are:

$$
\begin{aligned}
& \frac{\partial F_{G}}{\partial \theta}-\frac{d}{d z}\left(\frac{\partial F_{G}}{\partial \theta^{\prime}}\right)=0, \\
& \frac{\partial F_{G}}{\partial \varphi}-\frac{d}{d z}\left(\frac{\partial F_{G}}{\partial \varphi^{\prime}}\right)=0 .
\end{aligned}
$$

This tedious task can be easily solved with symbolic software, e.g. MUpad (MATLAB) or MAPLE. In the MAPLE case, the software has implicit functions that solve the Euler-Lagrange equations with only two lines of code:

$>>E L:=$ remove(has, EulerLagrange $(\mathrm{Wg}, \mathrm{z},[\theta(\mathrm{z}), \varphi(\mathrm{z})]), \mathrm{K})$

>>(Thetadif,Phidif):=selectremove(has,EL,diff(diff(theta(z),z ),z)) 
The result is the Eqs. (8) expanded. To isolate the second order differential parameter and achieve the following functions,

$$
\begin{aligned}
& \theta^{\prime \prime}(z)=f\left(\theta(z), \varphi(z), \theta^{\prime}(z), \varphi^{\prime}(z)\right), \\
& \varphi^{\prime \prime}(z)=f\left(\theta(z), \varphi(z), \theta^{\prime}(z), \varphi^{\prime}(z)\right) .
\end{aligned}
$$

These equations relate the tilt and twist angles and the applied voltage with the position, being the key to solve the NLC director orientation by numerical methods. In order to obtain Eqs. (9), a simple instruction is used, solve:

>>solve(Thetadif, $\operatorname{diff}(\operatorname{diff}(\theta(\mathrm{z}), \mathrm{z}), \mathrm{z}))$ )

$$
\frac{d^{2} \theta(z)}{d z^{2}}=\cos (\theta(z)) \sin (\theta(z))\left(\frac{A}{K_{33}+K_{11} \cos ^{2}(\theta(z))-K_{33} \cos ^{2}(\theta(z))}\right),
$$

with

$$
\begin{aligned}
A=K_{11}\left(\frac{d \theta(z)}{d z}\right)^{2}+2 K_{22} & \frac{d \varphi(z)}{d z} P-2 K_{22} \cos ^{2}(\theta(z))\left(\frac{d \varphi(z)}{d z}\right)^{2}- \\
& -K_{33}\left(\left(\frac{d \varphi(z)}{d z}\right)^{2}+\left(\frac{d \theta(z)}{d z}\right)^{2}-2 \cos ^{2}(\theta(z))\left(\frac{d \varphi(z)}{d z}\right)^{2}\right)-\left(\frac{d V(z)}{d z}\right)^{2} \Delta \varepsilon,
\end{aligned}
$$

>>solve(Phidif, diff(diff( $\varphi(\mathrm{z}), \mathrm{z}), \mathrm{z}))$ )

$$
\frac{d^{2} \varphi(z)}{d z^{2}}=\frac{2 \sin (\theta(z)) \frac{d \theta(z)}{d z} \frac{d \varphi(z)}{d z}\left(-2 K_{33} \cos ^{2}(\theta(z))-K_{22} P+K_{33}+2 K_{22} \cos ^{2}(\theta(z))\right)}{\cos (\theta(z))\left(K_{33}+K_{22} \cos ^{2}(\theta(z))-K_{33} \cos ^{2}(\theta(z))\right)}
$$

In parallel, the electrical field change across the cell, as a function of the anisotropy, must be taken into account. For potential profile estimation, Gauss's law is solved:

$$
\nabla \cdot \vec{D}=0 \text {. }
$$

Electrical displacement can be expressed by the applied potential (V), see Eq. (3). Then, considering the permittivity tensor in $\mathrm{z}$ direction (Eq. (4)) and isolating the second order differential equation of electrical field $V$, the code is as follows:

$>$ ez:=eo+ince $(\mathrm{n}[3])$

>>solve(diverge $(\mathrm{e} 0 \cdot \mathrm{ez} \cdot \mathrm{E},[\mathrm{z}]), \operatorname{diff}(\operatorname{diff}(\mathrm{V}(\mathrm{z}), \mathrm{z}), \mathrm{z}))$ )

$$
\begin{aligned}
& \frac{d^{2} V(z)}{d z^{2}}= \\
& -\frac{2 \Delta \varepsilon \cdot \sin (\theta(z)) \cos (\theta(z)) \frac{d \theta(z)}{d z} \frac{d V(z)}{d z}}{\varepsilon_{o}+\Delta \varepsilon \cdot \sin ^{2}(\theta(z))} .
\end{aligned}
$$

Finally, we have a system of three second order differential equations $\left(\theta^{\prime \prime}, \varphi^{\prime \prime}, V^{\prime \prime}\right)$, Eqs. (10), (12), and (14). This system has two boundary conditions, corresponding to the two glass plate voltages, then, it can be expressed as:

$$
Y^{\prime \prime}=f\left(z, Y, Y^{\prime}\right) ; Y(0)=\alpha ; Y(d)=\beta .
$$

where $\alpha$ and $\beta$ are vectors $(3 \times 1)$ with $Y$ solutions at the extreme points 0 and $d$, respectively. The analytical resolution is very complex, but numerical methods can define approximations to the solution. The latter equation, as well as being a boundary value problem (BVP), is non-linear reducing the possible methods to solve it.

\section{2.b. Proposed algorithm}

Firstly, the algorithm transforms the BVP into an initial value problem.

$$
Y^{\prime \prime}=f\left(z, Y, Y^{\prime}\right) ; Y(0)=\alpha ; Y^{\prime}(0)=v .
$$

The value of $\alpha$ is a $3 \times 1$ vector with the applied voltage $(V)$, the pretilt angle $\left(\theta_{1}\right)$ and the twist angle $\left(\varphi_{1}\right)$. The value of the boundary condition, $v$, that is also a vector, has to be determined for $Y^{\prime}$. A simple algorithm can do this, by a trialerror iterative process, starting with one arbitrary $v$ value, and solving the initial problem in the $[0, d]$ range. If the solution is $Y(d)=\beta$, the estimated initial condition $(v)$ is right, if not, the process starts again. However, this simple method is very inefficient. So, an improvement 
has been made by solving the problem of the unknown initial condition as a root of the following equation:

$$
r(v)=g(v)-\beta=0,
$$

where $r$ is the residual, that is, the difference between the estimation of the solution in d (with the initial condition $v$ ) and the solution in $d(\beta)$. This equation has been solved with the NewtonRaphson method, because it is simpler and faster than other methods such as the Brent or bisection algorithm. In addition, it is the best method for nonlinear equations. The differential equations are solved by an adaptive fourth order Runge-Kutta algorithm. The three second order equations system has been transformed to a six first order equations system by substitution $\left(y_{1}=\theta, y_{2}=\theta^{\prime}, y_{3}=\varphi, y_{4}=\varphi^{\prime}, y_{5}=V, y_{6}=V^{\prime}\right)$ in order to be able to use the Runge-Kutta algorithm.

$$
F(z, y)=Y^{\prime}=\left[\begin{array}{l}
y_{1}^{\prime} \\
y_{2}^{\prime} \\
y_{3}^{\prime} \\
y^{\prime}{ }_{4} \\
y_{5}^{\prime} \\
y_{6}^{\prime}
\end{array}\right]=\left[\begin{array}{c}
y_{2} \\
\theta^{\prime \prime} \\
y_{4} \\
\varphi^{\prime \prime} \\
y_{6} \\
v^{\prime \prime}{ }_{1}
\end{array}\right] .
$$

The complete algorithm, to solve the system, is depicted in the Fig. 3, and it is described by the following steps:

1. Two initial conditions for each equation of the system are set. For the $\theta^{\prime \prime}$ equation, the pretilt; for $\varphi^{\prime \prime}$, the twist, and for $V / N$, the voltage corresponding to the current step. For the unknown parameters $\theta^{\prime}, \varphi^{\prime}, V^{\prime}$ an approximation is made: $\theta^{\prime}=$ pretilt, $\varphi^{\prime}=$ totaltwist $/ d_{L C}, \quad$ and $\quad V^{\prime}=$ volts $/ d_{L C}$, ( $d_{L C}=$ sample thickness).

2. The initial condition $v$ takes the value of the previous $Y^{\prime}(0)$.

3. Eq. (17) is solved by the Newton-Raphson method, giving the initial condition, $v$, which produces the minimum residual, $r(v)$.

4. Once these values are known the differential equations are solved and the results are obtained.

5. The step, $N$, is reduced by one, such that voltage is increased one portion.

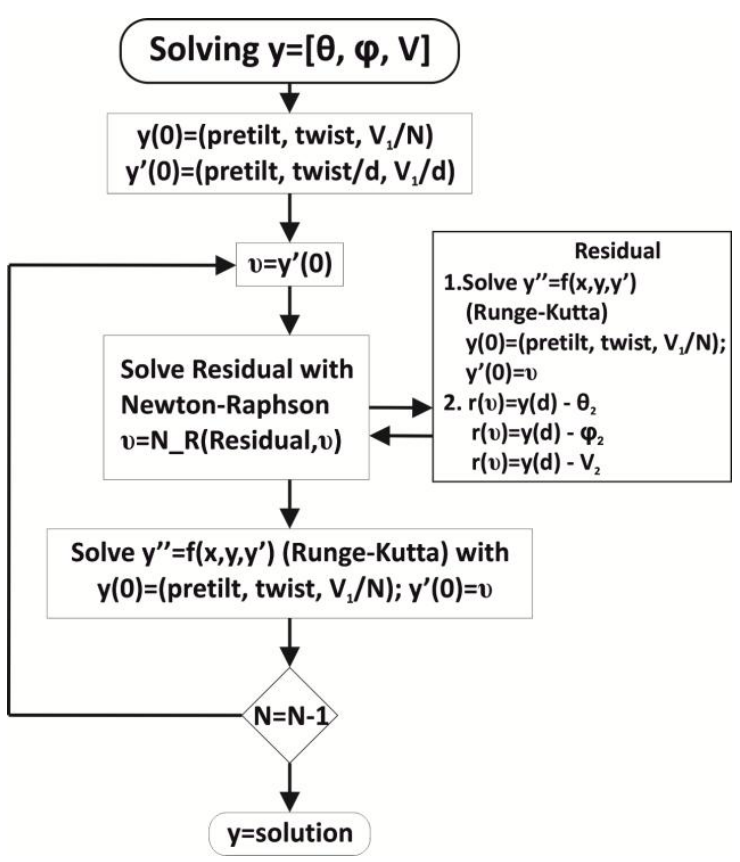

Fig.3. Algorithm flow chart.

6. The process is repeated until $N=1$ and the initial voltage has its final value. For this voltage the solution is $\theta(z), \varphi(z), V(z)$.

The algorithm solves the two angles $\theta, \varphi$ and the voltage across the dimension $z$. With these solutions, parameters such as birefringence or permittivity can be easily estimated. Once the angles are known, the estimation of the effective permittivity in each position can be made with the following equation:

$$
\varepsilon=\varepsilon_{o} \cos ^{2}(\theta)+\varepsilon_{e} \sin ^{2}(\theta) .
$$

To determine the permittivity as a function of voltage an integral is made:

$$
\left\langle\varepsilon_{e f f}\right\rangle(V)=\frac{1}{d} \int_{0}^{d} \varepsilon_{e f f}(V, z) d z .
$$

For the effective refractive index, the previous angles are also considered [18]:

$$
n_{e f f}(V)=\frac{1}{\sqrt{\frac{\sin ^{2}(\theta)}{n_{e}^{2}}+\frac{\cos ^{2}(\theta)}{n_{o}^{2}}}} .
$$

And finally, the birefringence can be easily solved by integration [19]: 


$$
\left\langle n_{e f f}\right\rangle(V)=\frac{1}{d} \int_{0}^{d} n_{e f f}(V, z) d z .
$$

The predicted parameters can be compared with measurements in a monopixel cell. In addition, they can be used for other devices if the voltage at different positions is known.

\section{Comparison with previous methods}

The proposed algorithm has been compared with two other methods: the classic FDM, with central approximation, and the proprietary algorithm of MATLAB, Bvp4c. As noted above, the last algorithm is known for its minimal error in BVP solutions. So, the comparison between the three methods has been done in terms of computational cost and errors, taking as reference the Bvp4c results. A device with parallel alignment has been chosen for the comparison; this configuration is one of the most used in the newest experimental applications (optical filters, microwave devices, active zoom, etc.). The twist angle is $0^{\circ}$ and the elastic constant $K_{22}$ is not taken into account. The LC chosen is MDA-98-1602 (Table I) with a device of $6.5 \mu \mathrm{m}$ thickness and $5^{\circ}$ of pretilt caused by the alignment.

The previous parameters are considered for a $589.3 \mathrm{~nm}$ wavelength and $1 \mathrm{kHz}$ frequency $\mathrm{AC}$ electrical signal and are extracted from the datasheet provided by Merck. The three algorithms have been programmed in MATLAB. The calculation of the voltage and the angle profiles has been made with: 20 steps for the shooting and Bvp4c methods and an incremental number $\left(10^{4}-10^{2}\right)$ for the FDM method. Both profiles are shown in Fig. 4. It is advertised that, for different voltages $(2 \mathrm{~V}, 4 \mathrm{~V}$ and $6 \mathrm{~V})$, the number of errors is very small to distinguish between methods in the graphs.

The maximum tilt angle estimated is also similar between algorithms (Table II). However, the CPU time consumption has been measured on the basis of the three alternative methods of simulation (Table III). The results demonstrate that the proposed algorithm is faster than Bvp4c and FDM (for low voltages), with the accuracy of
TABLE I

MDA-98-1602 nematic LC characteristics

\begin{tabular}{c|c|c}
\hline \hline $\begin{array}{c}\text { Elastic } \\
\text { Constants }\end{array}$ & Birefringence & Permittivity \\
\hline \hline$K_{11}=15.7 \mathrm{pN}$ & $n_{e}=1.7779$ & $\varepsilon_{e}=16.2$ \\
$K_{22}=8 \mathrm{pN}$ & $n_{o}=1.5113$ & $\varepsilon_{o}=12$ \\
$K_{33}=13.6 \mathrm{pN}$ & $\Delta n=0.2666$ & $\Delta \varepsilon=4.3$ \\
\hline \hline
\end{tabular}
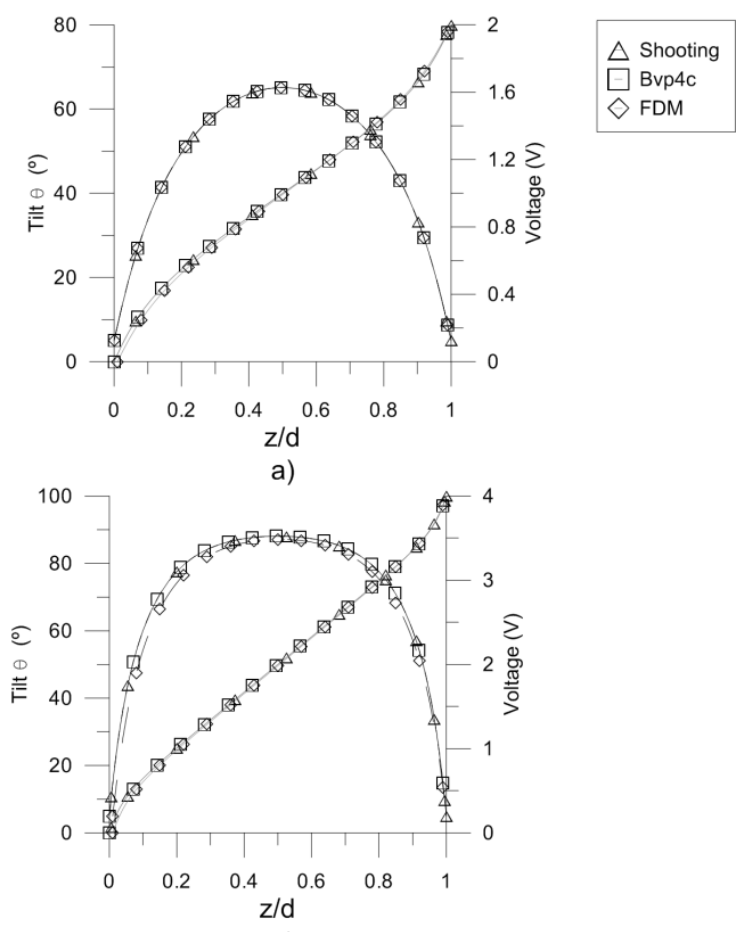

b)

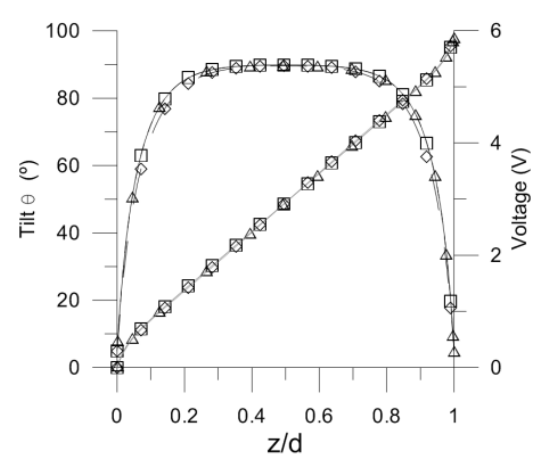

c)

Fig. 4. Simulations of LC director with three different methods: a) $2 \mathrm{~V}$, b) $4 \mathrm{~V}$ and c) $6 \mathrm{~V}$.

the proprietary algorithm of MATLAB, Bvp4c. FDM shows good behavior for high voltages, when the critical angle is almost ninety degrees.

On the other hand, FDM has a clear problem to find the optimal step size. There is a critical compromise between error and time 
TABLE II

Voltage dependence of maximum tilt angle estimation

\begin{tabular}{|c|c|c|c|}
\hline \hline Voltage & Shooting & Bvp4c & FDM \\
\hline \hline 2 Vrms & $65.11^{\circ}$ & $65.12^{\circ}$ & $61.55^{\circ}$ \\
4 Vrms & $88.10^{\circ}$ & $88.10^{\circ}$ & $87.14^{\circ}$ \\
6 Vrms & $89.93^{\circ}$ & $89.95^{\circ}$ & $89.73^{\circ}$ \\
\hline \hline
\end{tabular}

TABLE III

Voltage dependence of CPU time consumption

\begin{tabular}{|c|c|c|c|}
\hline \hline Voltage & Shooting & Bvp4c & FDM (Steps) \\
\hline \hline 2 Vrms & $2.09 \mathrm{~s}$ & $6.55 \mathrm{~s}$ & $6 \mathrm{~s}\left(5 \times 10^{4}\right)$ \\
4 Vrms & $1.80 \mathrm{~s}$ & $7.53 \mathrm{~s}$ & $1.8 \mathrm{~s}\left(10^{4}\right)$ \\
6 Vrms & $2.60 \mathrm{~s}$ & $7.37 \mathrm{~s}$ & $0.3 \mathrm{~s}\left(2 \times 10^{3}\right)$ \\
\hline \hline
\end{tabular}

consumption. The number of steps in the proposed algorithm is always 20 , demonstrating ease of use (there is no need to estimate the step for different supply voltages).

\section{Experimental setup}

In order to demonstrate the algorithm performance, a monopixel cell has been manufactured and characterized. The main parameters are: NLC MDA-98-1602, thickness $6.5 \mu \mathrm{m}$ and surface $5 \mathrm{~cm}^{2}$. Two different characterizations have been carried out. The process to obtain the birefringence is based on transmittance between parallel and crossed polarizer, measured by spectroscopy. The dielectric anisotropy is based on a Wheatstone bridge setup taking into account the electrical equivalent circuit at $1 \mathrm{kHz}$ (a resistance parallel capacitor).

\subsection{Birefringence}

One of the most important LC characteristics is its optical anisotropy or birefringence. This feature, which can be tuned by an external AC square voltage, has been chosen to validate the presented algorithm. For an experimental measurement the set up of Fig. 2 is employed.

The experimental set-up was comprised of an AvaSpec-128 fiber optic spectrometer from Avantes with $4 \mathrm{~nm}$ of resolution in a range of 360-890nm. Driving signal was a $1 \mathrm{kHz}$ AC square signal from 0.1 to $6 \mathrm{Vrms}$ (steps of 0.005 Vrms). The cell is supplied by a square alternating signal of $1 \mathrm{kHz}$, with RMS voltage from 0.1 to 6 Vrms with a resolution of 0.005 Vrms. Theoretically, optical transmittances between parallel $\left(I_{\|}\right)$and crossed polarizer $\left(I_{\perp}\right)$ are [20]:

$$
I_{\|}=I_{0} \cos ^{2}\left(\frac{\Phi}{2}\right), I_{\perp}=I_{0} \sin ^{2}\left(\frac{\Phi}{2}\right) .
$$

where $I_{0}$ is the intensity of the input light and $\Phi$ the phase shift. From Eq. (23), the phase shift can be extracted as:

$$
\Phi=\left\{\begin{array}{cc}
N_{m} \pi+\operatorname{arcos}\left(\frac{I_{\|}-I_{\perp}}{I_{\|}+I_{\perp}}\right) & N_{m}=0,2,4,6 \ldots \\
\left(N_{m}+1\right) \pi+\operatorname{arcos}\left(\frac{I_{\|}-I_{\perp}}{I_{\|}+I_{\perp}}\right) & N_{m}=1,3,5,7 \ldots
\end{array}\right.
$$

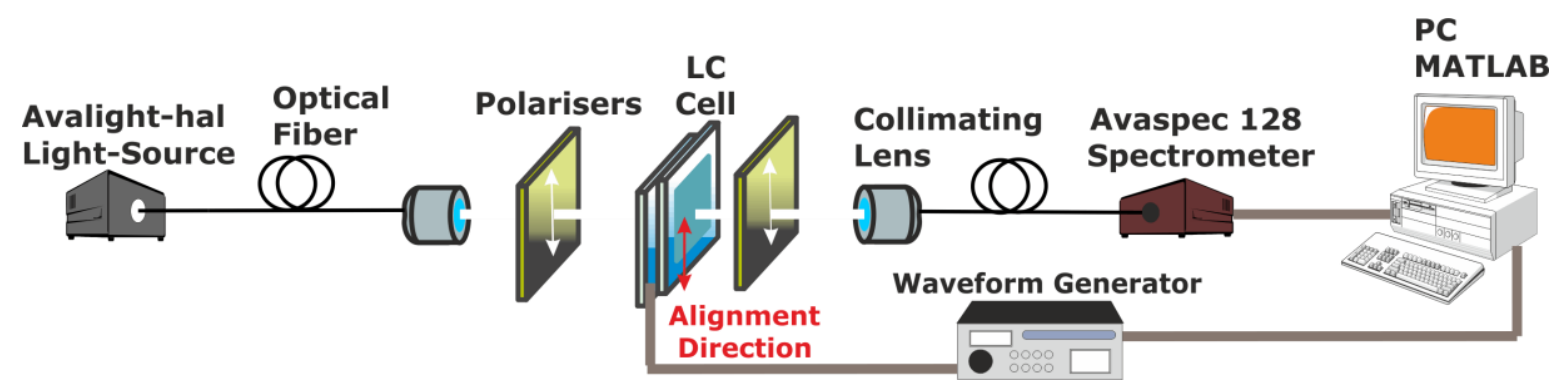

Fig. 5. Experimental set up for transmittance measures, between crossed and parallel polarizer. 
where $N_{m}$ is the number of maximums and minimums in the transmittance profile. The transmittance profiles are processed by MATLAB, particularly concerned with the calculus of the phase as a function of voltage following Eq. (24). The result is a set of birefringence values as a function of voltage for various wavelengths (dispersion).

\section{4.b. Dielectric anisotropy}

In order to characterize the dielectric response, impedance spectroscopy analysis is usually used. Notwithstanding, this technique is mainly valid for low voltages, when non-linear effects can be deprecated. In this case, and considering a specific frequency range where an equivalent electrical circuit of LC is known, a classic Wheatstone bridge is used.

This set-up is composed of the same LC cell previously used $\left(Z_{x}\right)$, placed in one branch. On the left side are the variable components $\left(Z_{1}\right)$ for tune purposes. In order to simplify the measurements, two equivalent impedances are placed in the lower branches (Eq. (25)). This is why $Z_{2}$ is a potentiometer. This bridge configuration produces null voltages between $\mathrm{BC}$ when the following equation is reached:

$$
Z_{x}=\frac{Z_{1} Z_{3}}{Z_{2}} .
$$

If all of the lower branch components are equal, $Z_{2}=Z_{3}$, the bridge is in equilibrium when $\mathrm{BC}$ voltage is equal to zero $\left(V_{d}=0\right)$, in other words, when $Z_{x}=Z_{1}$. As commented above, this EEC is valid only for a certain range of frequencies $(100$ $\mathrm{Hz}-10 \mathrm{kHz}$ ). In this case the EEC is simply a capacitor parallel to a resistance (dielectric losses). For better precision, an operational amplifier (AD620) with $\times 100$ factor gain is placed in BC points. The experiment take measurements of resistance and capacitance

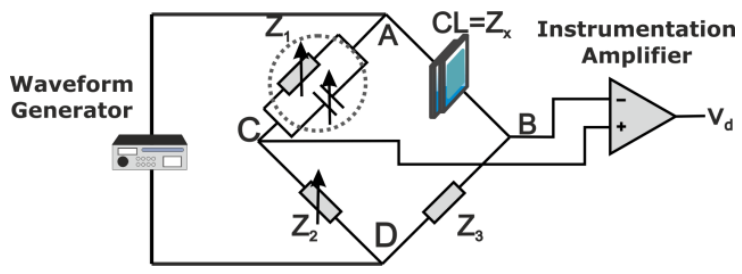

Fig. 6. Wheatstone bridge for measuring the LC dielectric permittivity of MDA-98-1602 in a voltage range.
$\left(Z_{1}\right)$ when $V_{d}=0$, for a range of voltages from 0 6 Vrms (frequency $=1 \mathrm{kHz}$ ).

\section{Experimental and simulated results}

The experimental results have been compared with simulations, of the same LC cell employed in these set-ups, using the proposed algorithm.

\section{5.a. Birefringence}

Following Eq. (21), the birefringence as a function of voltage can be determined. The voltage dependence of the effective birefringence of a monopixel cell, with the same characteristics as the experimental set-up, was simulated following the proposed algorithm. Experimental birefringence profiles were obtained, for a set of wavelengths, using the procedure described in section 4.A. Figure 7 shows the comparison between the simulated and experimental results of the LC cell birefringence.

Upon comparing experimental data to those obtained in simulation, we found extremely close results. For high voltages birefringence tend to zero due to the perpendicular position of the molecules with respect to the cell surface. When the voltage is lower than the threshold the molecules are aligned parallel to the surface and the birefringence is the highest. The model shows a small discrepancy for high voltages mainly caused by convergence problems when the angle tends to ninety degrees. Notwithstanding, these similar results validate the proper operation of the proposed algorithm.

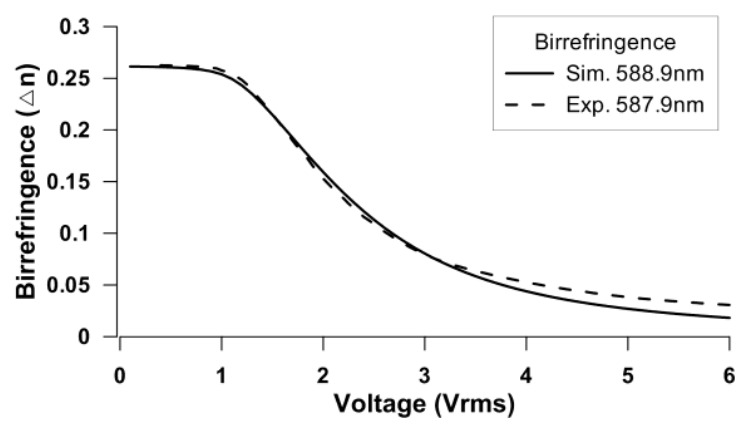

Fig. 7. Simulated and experimental birefringence as a function of voltage. 

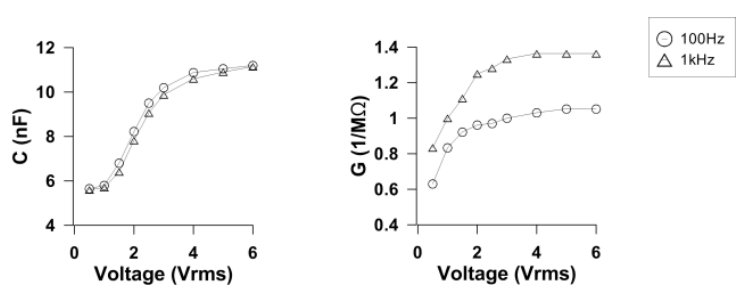

Fig. 8. Capacitance $(C)$ and conductance $(G=1 / R)$ of NLC MDA-98-1602 as a function of voltage and frequency.

\section{5.b. Dielectric anisotropy}

The result of the experimental set-up shown in Fig. 6 is the LC cell equivalent capacitance and resistance (Fig. 8).

The capacitance and conductance are increasing with voltage. This is caused by the homogeneous alignment of molecules, as in the case mentioned above. In this case the permittivity considered in the relaxed position is the ordinary one. With cell dimensions and classic capacitor equation, permittivity is easy to calculate from this measure. Once the permittivity is extracted, a simulation with the same cell parameters is carried out. Eq. (19) is used for effective permittivity estimation, and then, Eq. (20) is used for permittivity estimation as a function of voltage. These results are shown in Fig. 9.

This example also validates the behavior of the proposed algorithm. The slight differences between data could be caused by experimental measurement errors. In the case of imaginary permittivity extracted from conductance, the simulations cannot be carried out due to lack of this data in the LC data sheet.

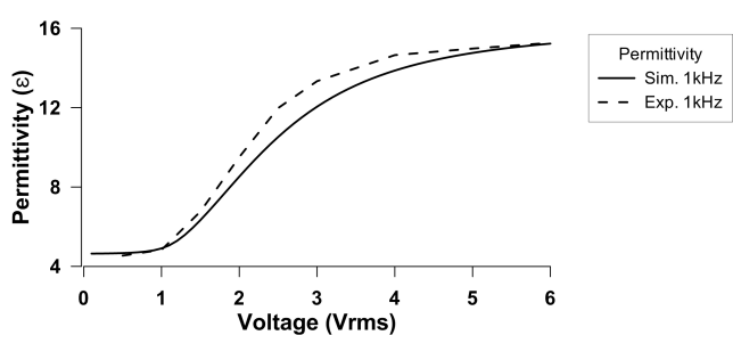

Fig. 9. Theoretical and experimental permittivity as a function of voltage.

\section{Conclusions}

The minimization of Gibbs free energy has been solved for a LC molecular arrangement. A novel, accurate, fast and reliable algorithm has been proposed and the initial prediction compared with experimental data has been presented. The comparison between experimental and simulated data shows close results. These simulations can be easily extended to other experimental devices where voltage distribution is known.

\section{Acknowledgements}

Acknowledgements: This work was supported in part by the Ministerio de Ciencia e Innovación of Spain (grant no. TEC2009-13991-C02-01) and Comunidad de Madrid (grant no. S2009/ESP1781). 